\title{
Streptomyces griseus Streptomycin Phosphotransferase: Expression of Its Gene in Escherichia coli and Sequence Homology with Other Antibiotic Phosphotransferases and with Eukaryotic Protein Kinases
}

\author{
By CHOR-KIANG LIM, $†$ MARGARET C. M. SMITH, JUNE PETTY \\ (NÉE HERCOMB), §SIMON BAUMBERG* AND JOHN C. WOOTTON \\ Department of Genetics, University of Leeds, Leeds LS2 9JT, UK
}

(Received 19 April 1989; revised 28 August 1989; accepted 11 September 1989)

\begin{abstract}
The aphD gene of Streptomyces griseus, encoding a streptomycin 6-phosphotransferase (SPH), was sub-cloned in the pBR322-based expression vector pRK9 (which contains the Serratia marcescens trp promoter) with selection for expression of streptomycin resistance in Escherichia coli. Two hybrid plasmids, pCKL631 and pCKL711, were isolated which conferred resistance. Both contained a $\sim 2 \mathrm{kbp}$ fragment already suspected to include aphD. The properties of in vitro deletion derivatives of these plasmids were consistent with the presumed location of $a p h D$. In vitro deletion of a sequence including most of the trp promoter largely, but not quite completely, abolished the ability of the plasmid to confer streptomycin resistance, confirming that expression was indeed principally from the trp promoter. A polypeptide of $\sim 34.5 \mathrm{kDa}$ was present in minicells containing plasmids that conferred streptomycin resistance, but was absent when the plasmids contained in vitro deletions removing streptomycin resistance. Part of the fragment was sequenced and an open reading frame corresponding to $a p h D$ identified. A computer-assisted comparison of the deduced SPH sequence with those of other antibiotic phosphotransferases suggested a common structure A-B-C-D-E, where B and D were conserved between all sequences compared while $\mathrm{A}, \mathrm{C}$ and $\mathrm{E}$ divided between the streptomycin and hygromycin B phosphotransferases on one hand and kanamycin/neomycin ones on the other. A composite sequence database was searched for homologues to consensus matrices constructed from five approximately 12-residue subsequences within blocks B and D. For one subsequence, corresponding to the $\mathrm{N}$-terminal portion of block $\mathrm{D}$, those sequences from the database that yielded the highest homology scores comprised almost entirely either antibiotic phosphotransferases or eukaryotic protein kinases. Possible evolutionary implications of this homology, previously described by other groups, are discussed.
\end{abstract}

\section{INTRODUCTION}

Antibiotic-producing streptomycetes carry one or more genes that render the producer strain resistant to its own antibiotic. Cloning of such genes is valuable for several reasons. First, an antibiotic resistance gene is often part of a cluster containing some or all of the genes for biosynthesis of the antibiotic and the control of their expression. Cloning of the resistance gene may thus provide a means of obtaining sequences involved in biosynthesis. Second, the regulation of expression of a resistance gene may be significant: in some cases it is only expressed together with the genes encoding enzymes of antibiotic synthesis under conditions of 'secondary metabolism', and the regulatory processes determining this pattern are in no case

\footnotetext{
† Present address: Department of Microbiology, National University of Singapore, Singapore 0511.

‡ Present address: Institute of Genetics, University of Glasgow, Glasgow G11 5JS, UK.

$\S$ Present address: Biotechnology Centre, UMIST, Manchester M60 1QD, UK.
}

Abbreviation: SPH, streptomycin phosphotransferase. 
well understood. Third, the evolutionary and functional relationships of resistance proteins from streptomycetes to each other, to similar proteins encoded by antibiotic resistance plasmids, and to further classes of proteins, are likely to be of interest. (For references see Hopwood et al., 1986; Hunter \& Baumberg, 1989.)

We have for these reasons cloned a gene encoding streptomycin phosphotransferase (SPH) activity from the streptomycin producer Streptomyces griseus ATCC 12475 (Vallins \& Baumberg, 1985). Five other groups have cloned SPH-encoding genes, four from strains of $S$. griseus (Tohyama et al., 1984; Distler \& Piepersberg, 1985; Distler et al., 1985; Ohnuki et al., 1985; Shinkawa et al., 1985) and one from a dihydrostreptomycin-producing strain of $S$. glaucescens (Hintermann et al., 1984). Distler et al. (1985) showed that their strain of $S$. griseus contains two distinct genes that encode enzymes with SPH activity. From the published restriction maps and sequence data (see also below), it may be deduced that the genes cloned by Tohyama et al. (1984), Ohnuki et al. (1985) and Vallins \& Baumberg (1985) correspond to one of the genes of Distler et al. (1985), and that cloned by Shinkawa et al. (1985) to the other. Distler $e t$ al. (1985) and Ohnuki et al. (1985) showed that the enzyme encoded by the first gene phosphorylates streptomycin at position 6 of its streptidine moiety; the same activity was demonstrated for the product of the cloned $S$. glaucescens gene by Hintermann et al. (1984). These enzymes are therefore of the APH(6) type. Shinkawa et al. (1985) suggested that the second $S$. griseus gene also encodes an APH(6), but more recently Heinzel et al. (1988) have provided cogent evidence that this enzyme is a streptomycin 3"-phosphotransferase, or more generally an APH(3"). Sequences for the first $S$. griseus gene (Tohyama et al., 1987; Distler et al., $1987 a$ ), the second $S$. griseus gene (Shinkawa et al., 1987; Heinzel et al., 1988) and the $S$. glaucescens gene (Vögtli \& Hütter, 1987) have been published. The sequences of Tohyama et al. (1987) and Distler et al. (1987a) are very similar (see also below), and show strong (>70\%) homology to that of Vögtli \& Hütter (1987). We will here follow Distler et al. (1987a) and Vögtli \& Hütter (1987) in referring to their SPH[APH(6)]-encoding genes as $a p h D$ and $s p h$ respectively. The gene cloned in our laboratory is therefore an aphD gene. aphD has been shown (Ohnuki et al., 1985; Distler et al., 1985, 1987b; Tohyama et al., 1987) to be part of a cluster containing genes of streptomycin biosynthesis and its regulation. The sequences for the second $S$. griseus gene reported by Shinkawa et al. (1987) and Heinzel et al. (1988) differ appreciably; it appears (see Shinkawa et al., 1988) that the latter's version is to be accepted. We shall follow Heinzel $e t$ al. (1988) in referring to this gene as aphE.

It is well known that expression of a Streptomyces gene in Escherichia coli usually requires its placing close to and downstream of an active vector promoter (suggesting that failure of expression in other circumstances may reflect inability of the $E$. coli RNA polymerase to recognize some Streptomyces promoters). Formal proof that transcription is initiated at the vector promoter is lacking in most cases, but has been provided in the case of a cloned leuA gene from a strain of $S$. rochei (Hercomb et al., 1987). It would be advantageous to develop more generally applicable systems for expression of Streptomyces genes in E. coli: this would for instance facilitate isolation of those genes with functional $E$. coli analogues by complementation of appropriate mutants.

Genes from various sources encoding a number of phosphotransferases active on aminoglycoside antibiotics other than streptomycin have been cloned and sequenced. These include kanamycin/neomycin 3'-phosphotransferases from several Gram-positive and Gramnegative organisms (Herbert et al., 1986), aminoglycoside 6'-acetyltransferase 2"-phosphotransferase from Streptococcus faecalis (Ferretti et al., 1986) and hygromycin B phosphotransferase from Streptomyces hygroscopicus (Zalacain et al., 1986). Comparison of the deduced amino acid sequences demonstrate that these all show homology in varying degrees to the enzymes encoded by $S$. griseus aphD and $S$. glaucescens sph, as well as to each other. These relationships were described by Distler et al. (1987a), who furthermore suggested that, remarkably, all these enzymes contain two regions with homology to separate regions within vertebrate serine/ threonine protein kinases, one of these regions also showing homology to tyrosine protein kinases including those encoded by proto-oncogenes. A similar relationship of the bacterial antibiotic phosphotransferases to vertebrate protein kinases has also been noted by Brenner 
(1987), who however found only one homologous region within which the homology was indicated to be more extensive than that proposed by Distler et al. (1987a). Heinzel et al. (1988) extended the comparison between antibiotic phosphotransferases and protein kinases, and described four regions of homology: an $\mathrm{N}$-proximal region $\mathrm{G} / \mathrm{K}$ whose sequence has features suggesting involvement in ATP binding; regions I and II, corresponding to the first and second regions in Distler et al. (1987a); and region III, slightly downstream from region II. The extended region of homology described by Brenner (1987) corresponds to regions II and III of Heinzel et al. (1988) together. Piepersberg's group has recently published (Piepersberg et al., 1988) interesting proposals on the possible evolutionary origins of antibiotic-modifying enzymes.

We report here expression of our previously cloned $a p h D$ gene in $E$. coli following sub-cloning adjacent to the Serratia marcescens trp promoter in the vector pRK9, and demonstrate that aphD expression is dependent on the integrity of that promoter. We also report its sequence; a comparison of the deduced amino acid sequence of the encoded SPH enzyme with other antibiotic phosphotransferases; and the results of a computer search for proteins sharing regions of homology with these phosphotransferases.

\section{METHODS}

Strains, plasmids and growth conditions. E. coli K12 host strain XS1D2 has been described (Mann et al., 1984). The minicell-producing strain DS410 was provided by D. Sherratt (Institute of Genetics, University of Glasgow, UK). The origin and properties of hybrid plasmid pBV4, which carries an $11.55 \mathrm{kbp}$ insert of $S$. griseus chromosomal DNA cloned into the BgIII site of pIJ702, are described in Vallins \& Baumberg (1985). The vector pRK9 (Schechtman \& Yanofsky, 1983) is pBR322 with the 380 bp EcoRI-BamHI fragment replaced by a 95 bp EcoRI-BamHI fragment containing the Serratia marcescens trp promoter (Nichols \& Yanofsky, 1983). Streptomycin-containing liquid or solid media for $E$. coli work consisted of nutrient broth (Oxoid no. 2). Plasmidcontaining cells were grown up for plasmid extraction under appropriate antibiotic selection. E. coli cells in or on streptomycin media were incubated at $30^{\circ} \mathrm{C}$ unless otherwise stated.

DNA manipulation. pBV4 was prepared by the method of Kieser (1984). E. coli plasmids were prepared essentially by the method of Ish-Horowicz \& Burke (1981). Other in vitro DNA manipulations, including restrictions, ligations, $\mathrm{S} 1$ nuclease treatment and agarose gel electrophoresis, were as in Maniatis et al. (1982) or as recommended by the enzyme supplier. Gels were photographed with UV illumination (TL33 transilluminator, Ultraviolet Products Inc.) on Polaroid 665 film with a Kodak Wratten filter no. 15.

Probe preparation, Southern blot hybridizations, and tests for SPH activity. These were carried out as described by Vallins \& Baumberg (1985).

Preparation and in vivo labelling of minicells. These techniques were as described by Reeve (1979).

SDS-polyacrylamide gel electrophoresis. SDS-PAGE of minicell protein radiolabelled in vivo with $\left[{ }^{35}\right.$ S]methionine was carried out according to the method of Laemmli (1970). Radioisotope-labelled polypeptide bands were visualized by fluorography with 2,5 -diphenyloxazole.

Sub-cloning and sequencing procedures were as described in Smith et al. (1986). Sequencing was generally by the dideoxy chain termination method; the Maxam and Gilbert chemical method was employed for one fragment to overcome a compression problem caused by a highly G-rich region.

Computer analysis: sequence alignment and database exploration. Alignments of multiple amino acid sequences were constructed essentially by methods previously described (Drummond \& Wootton, 1987; Davison et al., 1989). Pairwise optimal alignments were made by a modification of the method of Smith \& Waterman (1981) using a variant of the PAM 250 mutational matrix in which scores for exact matches were boosted by a factor of 1.5 and gap penalties for indels of length $1,2,3$ and $>3$ were in the ratio $-1,-1.2,-1.33$ and -1.4 respectively. Pairwise alignments were combined manually into multiple alignments, using program MANALIGN of the LUPES software (Akrigg et al., 1988), by making minor adjustments in the positions of gaps necessary to minimize the number of gap boundaries in the overall alignment. Quality of alignments was assessed by statistical comparison with random sequences (Drummond \& Wootton, 1987) and also by the extent to which regions of similar secondary structure predictions were in register (joint consensus method of Eliopoulos et al., 1982, as modified by Davison et al., 1989).

Exploration of the composite amino acid sequence database OWL (Bleasby \& Wootton, 1989) used LUPES software employing feature-specific scoring matrices (Akrigg et al., 1988). Programs PATSCAN and MEGASCAN were used, essentially as previously described (Drummond \& Wootton, 1987; Wootton \& Drummond, 1989). The initial database scans used weight matrices constructed from the residue frequencies in subsequences of the multiple sequence alignment. Experimental modification of the weight matrices, using program CAM (N. I. M. Dix, J. Swift 
\& J. C. Wootton, unpublished), was employed for iterative database exploration, using similar rules and constraints to those previously outlined (Wootton \& Drummond, 1989). This process indicated the regions of the phosphotransferases that were most clearly homologous to each other and distinct from other proteins, and also the regions most strongly similar to the protein kinases. The LUPES programs SHOWTVF and HITRELATE (N. I. M. Dix, J. Swift \& J. C. Wootton, unpublished) were used to assess the power of the different weight matrices to discriminate the sequence features of interest (for example, regions of the phosphotransferases, subclasses of phosphotransferases, protein kinases or kinases plus phosphotransferases) from other families of homologous proteins in the OWL database.

\section{RESULTS AND DISCUSSION}

Sub-cloning of aphD with expression in E. coli and characterization of plasmids containing all or part of it

The hybrid plasmid pBV4 (Vallins \& Baumberg, 1985) comprises an $11.55 \mathrm{kbp}$ Sau3A insert of $S$. griseus ATCC 12475 DNA cloned into pIJ702; it confers streptomycin resistance and SPH activity in S. lividans. A partial Sau3A digest of pBV4 was ligated to BamHI-cleaved pRK9, and the ligation mixture used to transform the $E$. coli $\mathrm{K} 12$ sub-strain XS1D2 (which has a particularly low basal streptomycin resistance). Of 322 ampicillin-resistant transformants, nine were also resistant to $10 \mu \mathrm{g}$ streptomycin $\mathrm{ml}^{-1}$. Plasmids pCKL631 and pCKL711 isolated from two of these were characterized further.

Restriction maps of these plasmids and pBV4, deduced from fragment sizes following single and double digests with a variety of restriction endonucleases, are shown in Fig. $1(a, b, g)$. The inserts in pCKL631 and pCKL711 are of 13.0 and $6.3 \mathrm{kbp}$ respectively. It is seen that pCKL631 and pCKL711 both contain the $\sim 2 \mathrm{kbp}$ pBV4 fragment bounded by restriction sites $B g I I I / S a u 3 A(1)$ and BamHI(4), which was earlier concluded to contain the aphD gene (Vallins \& Baumberg, 1985). This fragment is oriented similarly in the two plasmids with respect to the vector, consistent with expression of $a p h D$ by readthrough from the latter's $\operatorname{trp}$ promoter. Colinearity between the restriction maps of the sub-cloned fragment and the insert in pBV4 breaks down immediately (pCKL631) or a little way (pCKL711) beyond their BamHI(2) site, corresponding to the BamHI(4) site of pBV4 (see Fig. 1), suggesting that the inserts in pCKL631 and pCKL711 are made up of several reassembled Sau3A fragments from pBV4. Southern blot hybridizations with ${ }^{32}$ P-labelled DNAs of pRK9, pCKL631, pCKL711, pIJ702 or pBV4 as probes were consistent with pCKL631 and pCKL711 carrying the above-mentioned $\sim 2 \mathrm{~kb}$ fragment from pBV4 adjacent to the trp promoter of pRK9 (results not shown).

To confirm the approximate extent of aphD, deletion derivatives of pCKL631 and pCKL711 were constructed. The two plasmids were completely digested with SstI and the products allowed to recircularize, giving rise to plasmids pCKL635 and pCKL719 respectively (Fig. $1 c$, $h$ ). Both plasmids conferred streptomycin resistance on $E$. coli XS1D2, confirming that most of the insert DNA in pCKL631 and pCKL711 is irrelevant to this phenotype. In addition, pCKL631 was completely digested with BamHI and the fragments allowed to religate under conditions intermediate between those favouring intramolecular recircularization and those favouring intermolecular ligation. Two new plasmids were obtained : pCKL633, which has lost pCKL631's BamHI(2)-BamHI(3) fragment but retains its BamHI(1)-BamHI(2) fragment, and pCKL634, which has lost both these fragments (Fig. 1 $d, e$ ). pCKL633 conferred streptomycin resistance but pCKL634 did not, confirming that the BamHI(1)-BamHI(2) fragment contains at least part of $a p h D$. Finally, the $B a m H I(1)-B a m H I(2)$ fragment was cloned into the BamHI site of pRK9, yielding pCKL636 (Fig. 1f). This plasmid did not confer streptomycin resistance, indicating (again in conformity with previous deductions) that part of $a p h D$ lies between pCKL631's BamHI/Sau3A(1) and BamHI(1) sites.

In summary, the results of restriction analysis, Southern blotting, construction of deletion derivatives, and assay of extracts for SPH activity are all consistent with a gene aphD encoding this activity being located within a $\sim 2 \mathrm{~kb}$ fragment, corresponding to $B g / \mathrm{II} / \mathrm{Sau} 3 \mathrm{~A}(1)$ BamHI(4) of pBV4 (Vallins \& Baumberg, 1985) or BamHI/Sau3A(1)-BamHI(2) or pCKL631 (Fig. 1). Data in both Vallins \& Baumberg (1985) and this paper are therefore consistent with the start and end of $a p h D$ lying respectively with pCKL631 fragments Bam HI/Sau3A(1)-BamHI(1) 
(a) $\mathrm{pBV} 4$

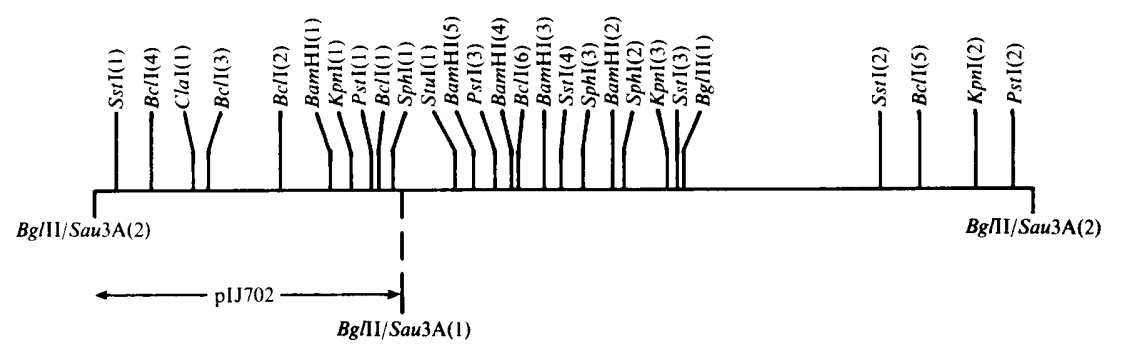

(b) pCKL.631

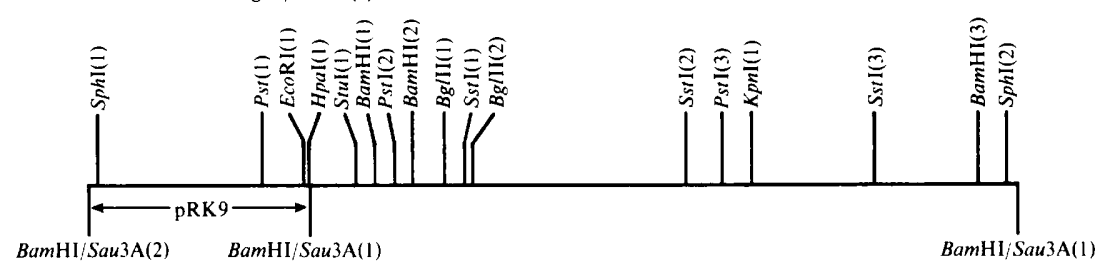

(c) pCKL635
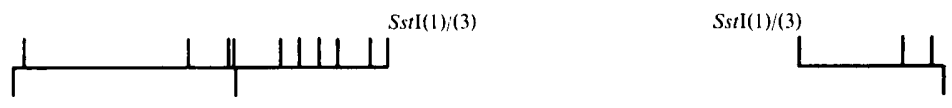

(d) pCKL633

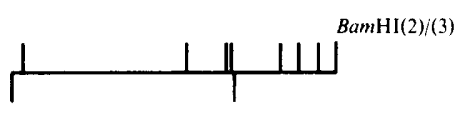

$\operatorname{BamHI}(2) /(3)$

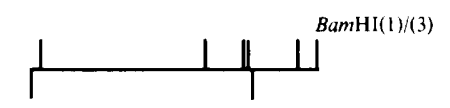

$\operatorname{BamHI}(1) /(3)$

(e) pCKL634

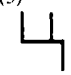

(f) pCKL636

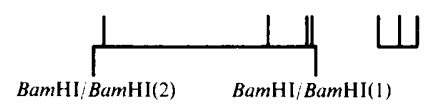

(g) pCKL711

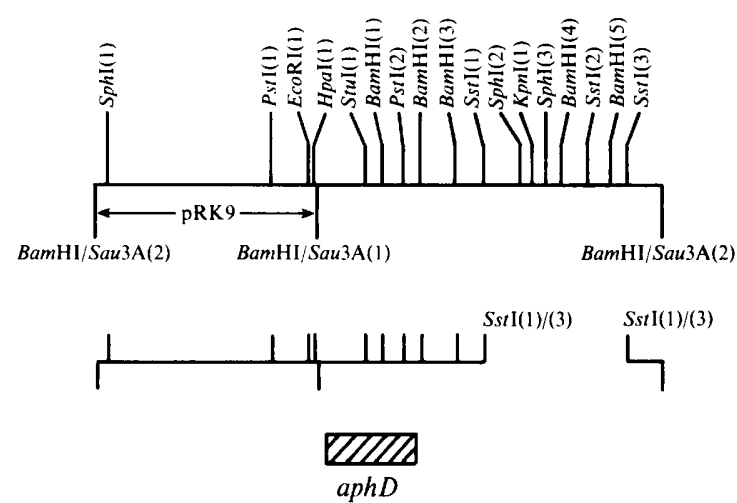

Fig. 1. Maps of pBV4, pCKL631, pCKL 711 and derived plasmids. The maps are aligned with constant vertical alignment of the vector/insert junction which lies just upstream of $a p h D$ and which is present in all these plasmids. The position of $a p h D$ is shown by a hatched block.

and PstI(2)-BamHI(2). Many of the details are not given in full since sequencing results (Tohyama et al., 1987; Distler et al., 1987a; see also below) confirm this interpretation.

Levels of streptomycin resistance conferred by the various plasmids in $E$. coli XS1D2 were determined at various growth temperatures. At $30^{\circ} \mathrm{C}$, isolates carrying pCKL631, pCKL633, pCKL635 and pCKL711 showed MICs of $30-40 \mu \mathrm{g} \mathrm{ml}^{-1}$, while those carrying pCKL719 

$-35$
$-10$
pRK9 AAAAAGAGGGTTGACTTTGCCTTCGCGAACCAGTTAACTAGTACACA

\section{pCKL631 AAAATACGCGTATCACGAGGCCCTTTCGTCTTCAAGAACTAGTACAC}

Fig. 2. Comparison of trp promoter sequence in pRK9 (and thereby in pCKL631 and pCKL711) and the predicted equivalent sequence in pCKL712 after in vitro deletion of the EcoRI-HpaI region. The underlined sequence is the $\mathrm{HpaI}$ site. The asterisk identifies the base remaining at the EcoRI-cleaved end after S1 treatment.

showed substantially higher MICs (about $70 \mu \mathrm{g} \mathrm{ml}^{-1}$ ). In all cases the resistance was much lower at $37^{\circ} \mathrm{C}$ and was more or less undetectable at $42{ }^{\circ} \mathrm{C}$. The reason for this phenomenon is not yet known. Protein extracts of cultures of XS1D2 carrying pCKL631, pCKL711 or pCKL719, but not pRK9, carried out ATP-dependent phosphorylation of streptomycin as estimated by the bacterial overlay assay (Vallins \& Baumberg, 1985) (results not shown), though activity was not detected using the same authors' coupled assay employing pyruvate kinase and lactate dehydrogenase.

\section{Effect on aphD expression of in vitro alteration of the vector trp promoter}

We wished to confirm that expression of $a p h D$ in $E$. coli is effected by the trp promoter, rather than by for instance a junction sequence with fortuitous promoter activity. Although expression of several streptomycete genes in $E$. coli by cloning in expression sites has been reported, only rarely has this control been included (see however Hercomb et al., 1987). pRK9 contains a unique HpaI site within the Serratia marcescens trp promoter (Fig. 2) overlapping the -10 region. The unique EcoRI site in pRK9 (the same site as in pBR322) comprises residues -76 to -71 , and the $H p a$ I site residues -14 to -9 , taking the trp-promoter-directed transcription start site as +1 (Nichols \& Yanofsky, 1983). pCKL711 DNA was cleaved with EcoRI, singlestranded ends removed by $\mathrm{S} 1$ nuclease digestion, and the products of this reaction cleaved with $H p a I$. The resulting mixture was then permitted to religate at the low DNA concentration of $10 \mu \mathrm{g} \mathrm{ml}^{-1}$ to favour intramolecular recircularization, and finally introduced into $E$. coli XS1D2 by transformation with selection for ampicillin resistance. Those isolates which proved also to be resistant to streptomycin $\left(10 \mu \mathrm{g} \mathrm{ml}^{-1}\right)$ contained plasmids with single $E c o \mathrm{RI}$ and $H p a \mathrm{I}$ sites, presumably pCKL711. Several isolates were obtained, however, which were sensitive to streptomycin at this concentration: all contained plasmids with no EcoRI or $\mathrm{HpaI}$ site. Further restrictions gave results consistent with all the latter plasmids being identical and resulting from deletion of the $54 \mathrm{bp}$ fragment between the EcoRI and HpaI sites of pCKL711. This construct was termed pCKL712. XS1D2(pCKL712) isolates showed an MIC of $4 \mu \mathrm{g} \mathrm{ml}^{-1}$ streptomycin, as opposed to XS1D2(pRK9), XS1D2(pCKL634) and XS1D2(pCKL636), which showed an MIC of $1 \mu \mathrm{g} \mathrm{ml}^{-1}$. The large reduction in streptomycin resistance, and hence presumably of $a p h D$ expression, was as expected if expression is directed principally by the trp promoter. The low residual expression may result from either or both of $(a)$ low remaining $\operatorname{trp}$ promoter activity - scrutiny of the pCKL712 sequence in Fig. 2 shows a conceivably functional -10 region, though no persuasive -35 region - or adventitious promoter functioning of vector sequences, or (b) slight activity in $E$. coli of a Streptomyces promoter.

This result confirms that with pCKL631 and pCKL711, as in other cases, expression has been attained by placing a fragment carrying a streptomycete gene downstream from, and in the correct orientation with respect to, a vector promoter. This was sufficient in the case of the $s p h$ gene (homologous to the aphD used here) from S. glaucescens ETH 22794 when sub-cloned downstream from the tet promoter of pBR322 (Hintermann et al., 1984). In the case of the plasmid pIJ401 of Hintermann et al. (1984), expression appears to have occurred when the start of the $s p h$ gene was at least $3.37 \mathrm{kbp}$ from the tet promoter. Decreasing this distance did not apparently increase the level of streptomycin resistance conferred. These authors used $2 \cdot 5-3 \mu \mathrm{g}$ 


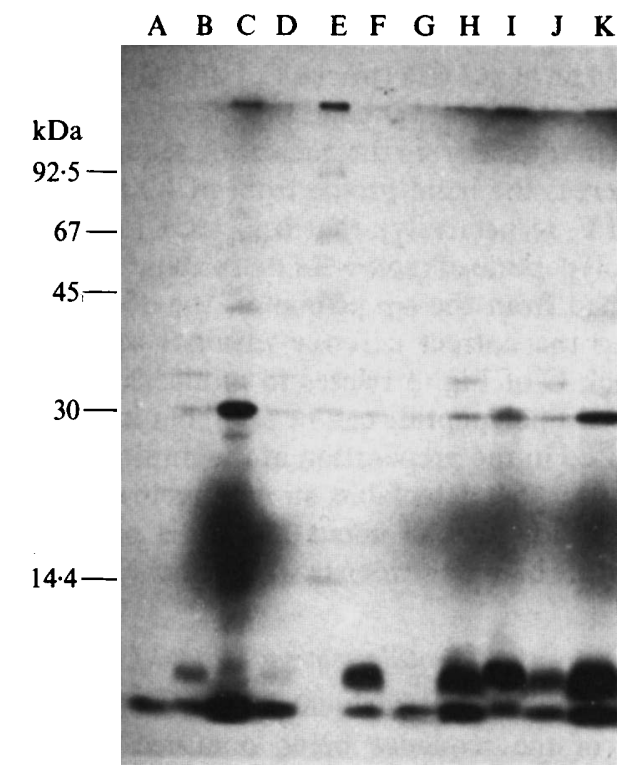

Fig. 3. Autoradiograph following SDS-PAGE of polypeptides, radiolabelled with $\left[{ }^{35}\right.$ S $]$ methionine, from minicells of $E$. coli DS410 without and with plasmids. Tracks: A, no plasmid; B, pCKL719; C, pCKL712; D, pCKL711; E, tritiated molecular mass standards - phosphorylase $b$ ( $92.5 \mathrm{kDa})$, bovine serum albumin $(67 \mathrm{kDa})$, ovalbumin $(45 \mathrm{kDa})$, carbonic anhydrase $(30 \mathrm{kDa})$ and $\alpha$-lactalbumin (14.4 kDa); F, pRK9; G, pCKL631; H, pCKL633; I, pCKL634; J, pCKL635; K, pCKL636.

streptomycin $\mathrm{ml}^{-1}$ for selection in $E$. coli, a considerably lower concentration than the $10 \mu \mathrm{g} \mathrm{ml}^{-1}$ employed here. Perhaps a temperature effect on the level of streptomycin resistance operated in their case as in ours (see above), since their incubations appear to have been carried out at $37{ }^{\circ} \mathrm{C}$. However, there are many other possible explanations for any difference in resistance observed in the two cases. More interestingly, in several instances a gene within a fragment cloned downstream from a vector promoter and in the correct orientation was not expressed until some of the intervening DNA had been deleted. This was found by Ohnuki et al. (1985) for the same gene as used here (their restriction map is identical to ours). When a $B g / I I$ fragment was cloned in the correct orientation downstream from the tet promoter of pBR322 (their plasmid pSMP2), separating the start of $a p h D$ from the tet promoter by about $3 \mathrm{kbp}$ (our estimation), there was no expression; but a deletion of about $2.4 \mathrm{kbp}$ of this intervening DNA enabled expression to occur. Comparable findings have been reported for a $p$-aminobenzoic acid synthetase gene from a candicidin-producing S. griseus (Gil \& Hopwood, 1983) and a cat gene from $S$. acrimycini (Gil et al., 1985). In these cases expression occurred only after deletion of about $1 \mathrm{kbp}$ and $0.7 \mathrm{kbp}$ of intervening DNA respectively. The implication appears to be that following the initial sub-cloning, transcription terminates within the region whose later deletion permits expression. Possibly streptomycete DNA has a high chance of containing sequences that mimic $E$. coli transcription stop signals.

\section{Characterization of the aphD gene product in minicells}

Plasmids pRK9, pCKL631, pCKL633, pCKL634, pCKL635, pCKL636, pCKL711, pCKL712 and pCKL719 were introduced into the minicell-forming strain DS410 by transformation with selection for ampicillin resistance. Minicells were prepared in each case and plasmid-encoded polypeptides labelled in vivo with L-[ $\left.{ }^{35} S\right]$ methionine. Labelled polypeptides were studied by SDS-PAGE followed by fluorography.

A band corresponding to a polypeptide of molecular mass $\sim 34.5 \mathrm{kDa}$ is seen in the autoradiograph in tracks from minicells containing pCKL631, pCKL633, pCKL635, pCKL711 or pCKL719 (Fig. 3, tracks G, H, J, D and B respectively), i.e. those plasmids that confer on $E$. 
coli XS1D2 resistance to $10 \mu \mathrm{g}$ streptomycin $\mathrm{ml}^{-1}$. This band is absent where the minicells contained pRK9, pCKL634 or pCKL636 (tracks F, I and K respectively). It is therefore likely that this polypeptide represents the $a p h D$ gene product. The estimated size is in reasonable accord with that of $33 \mathrm{kDa}$ predicted from the nucleotide sequence by Tohyama et al. (1987) and Distler et al. (1987a). Whereas the band profile from pCKL636 is effectively identical to that from pRK9 (tracks K and F, respectively), that from pCKL634 (track I) shows an additional band corresponding to a polypeptide of molecular mass about $30.5 \mathrm{kDa}$. This is again consistent with aphD being transcribed from the trp promoter, the $30.5 \mathrm{kDa}$ polypeptide then being a truncated version (lacking the correct carboxy-terminal sequence) of the $34.5 \mathrm{kDa}$ aphDencoded polypeptide. Track C of Fig. 3 relates to minicells containing pCKL712: the band corresponding to the $34.5 \mathrm{kDa}$ polypeptide can be seen, but is very faint even though about ten times more ${ }^{35} \mathrm{~S}$ label was used in the preparation of the minicells carrying this plasmid. This is consistent with the much lower, but detectable, streptomycin resistance conferred by pCKL712 (see above). Of the other bands, that of about $30 \mathrm{kDa}$ is presumably the vector-encoded $\beta$ lactamase. Differences in other bands between tracks probably do not relate to the present work.

\section{Nucleotide sequence of aphD}

The $\sim 1.9 \mathrm{kbp}$ Bg/II/Sau3A-BamHI fragment in pCKL631 shown above to contain aphD was sequenced, all parts of the sequence being obtained in both directions and with all restriction sites overlapped. The sequence obtained included the entire sequence comprising Fig. 2 of Distler et al. (1987a). There were no differences between our sequence and theirs. We conclude, in line with these authors and with Tohyama et al. (1987), who have also sequenced this region, that the major open reading frame within this sequence represents the SPH gene. This open reading frame starts with an ATG corresponding to nucleotide positions $+755 / 757$, taking the transcription start directed by pRK9's trp promoter as +1 , and encodes a 307-aminoacid polypeptide, reasonably consistent with the molecular mass of $34.5 \mathrm{kDa}$ proposed for SPH on the basis of the minicell experiments described above. Our sequence and that of Distler et al. (1987a), differ from that of Tohyama et al. (1987) in the following ways. The two base pair differences which give Gly in our sequence at position 225 as against Ala in that of Tohyama et al. (1987) are probably trivial: this would be a conservative change and might represent a genuine strain difference. The DNA sequences corresponding to the three differing blocks at amino acid positions 34-39, 115-120 and 181-189 differ only in the occurrence of $(+)$ and $(-)$ frameshifts (with, in the case of the last, a small number of further differences). All the amino acid differences occur in regions for which Tohyama et al. (1987) do not cite amino acid sequences of peptide fragments, which in several other regions amply confirm their DNA sequence. Further work will be necessary to resolve these inconsistencies. We will however use in what follows the amino acid sequence predicted from the DNA sequence obtained by Distler $e$ t al. (1987a) and by ourselves. We note in our sequence other features, such as a putative ribosome-binding site and both direct and inverted repeats, previously commented on by Tohyama et al. (1987) and/or Distler et al. $(1987 a)$.

\section{Relationships between the aphD product and other aminoglycoside phosphotransferases}

We have used a computer-assisted technique (see Methods) to align nine aminoglycoside phosphotransferase amino acid sequences. The enzymes were: $S$. griseus APH(6) (Distler et al., $1987 a$, and this paper); S. glaucescens SPH(6) (Vögtli \& Hütter, 1987); hygromycin B phosphotransferase from $S$. hygroscopicus (Zalacain et al., 1986); Tn5 streptomycin phosphotransferase, a presumed APH(6); and APH(3')s from $S$. fradiae, Tn5, Tn903, Bacillus circulans and Staphylococcus aureus (the last six compiled in Herbert et al., 1986). The results are shown in Fig. 4. In this alignment, regions of sequence similarity are arranged in a manner which is strongly suggestive of an interspersed functional map of the protein structure. In Fig. 4 the regions of sequence similarity are boxed and labelled A, B, C, D and E. Regions B and D are homologous in all the sequences, while regions $\mathrm{A}, \mathrm{C}$ and $\mathrm{E}$ divide between the streptomycin and hygromycin B phosphotransferases on the one hand (A1, C1 and E1) and the kanamycin/neo- 
mycin enzymes on the other (A2, C2 and E2). This interpretation is reached by the following reasoning.

The most strongly conserved region of the whole phosphotransferase family is region D (Fig. 4), which is also strongly similar in the protein kinase family (see below). This region was used as an initial anchor region for the multiple sequence alignment. From region D towards the Ctermini, the sequences clearly fall into two classes on grounds of length and sequence similarity, giving regions E1 and E2. Dynamic programming methods and attempts to align blocks of secondary structure predictions failed to give any significant matches between blocks E1 and E2, although on both criteria the matches within the E1 and E2 sets were strong.

Considering the sequences from region $\mathrm{D}$ towards the $\mathrm{N}$-termini, other blocks of strongly significant similarity, between all nine members of the phosphotransferase family, occur between positions 121 and 165 of the alignment of Fig. 4 (C-terminal portion of region B). These similarities, and also those in block $\mathrm{D}$, are strongly significant on grounds of statistical probability: all optimized pairwise comparisons give scores greater than three standard deviations from the mean pairwise alignment score using the modified PAM250 matrix described in Methods. However, the more convincing reason for regarding these regions as strongly homologous comes from the fact that the residue patterns in these regions effectively discriminate the phosphotransferase family from all other sequences in the OWL database of over 15000 proteins (Bleasby \& Wootton, 1989). Weight matrices were constructed, essentially by methods previously used (Drummond \& Wootton, 1987; Wootton \& Drummond, 1989; N. I. M. Dix, J. Swift \& J. C. Wootton, unpublished), from five short subsequences of the alignment. Those from region $\mathbf{D}$ and the $\mathbf{C}$-terminal section of region $\mathbf{B}$ (bars 1, 2, 3, 4 and 5 under the alignment, Fig. 4) were largely (but not in all cases completely) discriminating for aminoglycoside phosphotransferases (and protein kinases also in some cases: see below) when used with program MEGASCAN to search the whole OWL database. Since these strongly significant regions encompass extensive regions of the alignment, interspersed with less similar regions, we conclude that the enzymes are genuinely homologous in the sense of being related by divergence from a common ancestral sequence. Between blocks $B$ and $D$ the sequences cluster into two classes, $\mathrm{C} 1$ and $\mathrm{C} 2$, corresponding to the clusters of regions $\mathrm{E} 1$ and $\mathrm{E} 2$ described above.

The $\mathrm{N}$-terminal section of region $\mathrm{B}$ (alignment positions 50 to 120, Fig. 4) was then aligned on the basis of similarities to protein kinases (see below), rather than relying on possible homologies between the phosphotransferases themselves. Candidates for the known sequence features of the kinases that are involved in ATP-binding were present in all nine phosphotransferase sequences. Therefore this region is not divided into subsets of the sequences, but is included in the block B common to all nine. Alignment positions 1 to 49 (Fig. 4) show few conserved sequence features, but fall into two clusters, $\mathrm{A} 1$ and $\mathrm{A} 2$, largely on grounds of length, consistent with the division of the $C$ and $E$ regions.

It may be conjectured at this point that blocks $B$ and $D$ could represent domains concerned with ATP binding and phosphate transfer, while $\mathrm{A}, \mathrm{C}$ and $\mathrm{E}$ could represent domains involved in antibiotic recognition. A chemical interpretation of the division between the streptomycin/ hygromycin B and the kanamycin/neomycin phosphotransferases is that sequences common to the former are responsible for binding the 6-carbon rings (streptidine or hyoscamine) which are the phosphate acceptors in the reactions catalysed by these enzymes, whereas the sequences common to the kanamycin/neomycin phosphotransferases would be those responsible for recognizing the hexosamine rings which are the acceptors for the $\mathrm{APH}\left(3^{\prime}\right)$ reaction of these enzymes.

We have also carried out less complete studies with viomycin phosphotransferase (Bibb et al., 1985) and the APH(3") sequenced by Heinzel et al. (1988). These, although more distant, fall into the same pattern.

\section{Databank search for polypeptide sequences with homology to SPH and other aminoglycoside phosphotransferases}

To investigate the relationships of blocks $B$ and D of Fig. 4 to other proteins of known sequence, the OWL composite sequence database was searched by quantitative pattern 

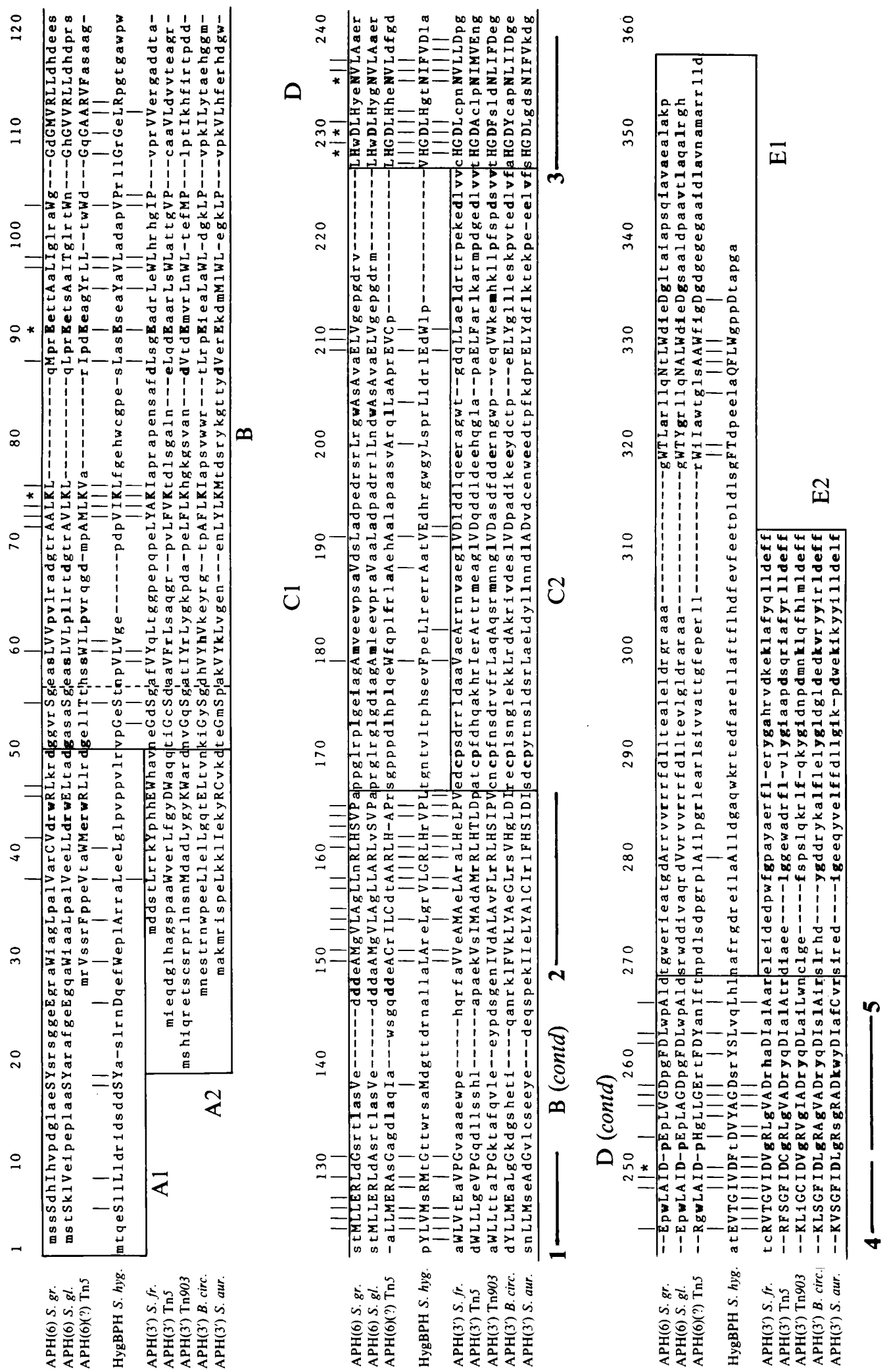
matching methods using consensus matrices (see Methods) constructed from the five different subsequences, from 7 to 17 residues in length, from within these blocks (see previous section and Fig. 4). Every protein sequence in the database yields a score for each matrix, high score corresponding to high similarity. The output of the search was a set of tabulations (not shown) of sequences in order of decreasing score, there being of course one tabulation for each of the five subsequences used to construct the matrices.

Examination of these tabulations revealed a particularly striking result in the case of subsequence 3, corresponding to the beginning of block D (positions 226-237 of the multiple alignment in Fig. 1), which contains the His, Asp and Asn residues that are completely conserved among the aminoglycoside phosphotransferases. Of the first 100 sequences in the tabulation for this matrix, the first eight were other bacterial aminoglycoside phosphotransferases; thereafter, all but five (nos 42, 60, 64, 96 and 98) of the remainder were vertebrate protein kinases of both the serine/threonine- and tyrosine-specific types. These included, in the order of their first appearance in the list (proto-oncogene products being referred to by the conventional gene designation): $e r b B$, phosphorylase $b$ kinase, neu, the Saccharomyces cerevisiae $c d c 2$ product, $s r c$, B-lym, yes, fgr, protein kinase C, kit, phs, raf, fms, mil, trk, ros, mos, fps, the insulin receptor, $a b l$, and fes. Many other protein kinases were in the 250 highest-scoring sequences in the tabulation for this matrix, but no other classes of ATP-binding proteins were consistently represented.

The indications provided by the other matrices were not as convincing. Subsequence 5 (corresponding to 13 residues towards the end of block D, Fig. 4) gave a possibly suggestive result in that of the 100 highest-scoring sequences, five were aminoglycoside phosphotransferases, two were protein kinases, and six were phosphoribosyltransferases or other ribonucleotidebinding proteins. The tabulations for subsequences, 1, 2 and 4 did not imply homology to any other class of proteins.

Despite the lack of close primary sequence homology between the phosphotransferases and protein kinases outside subsequence 3 , two further points of comparison emerge, particularly when structural considerations are additionally taken into account.

(i) The protein kinases show the characteristic ATP-binding site motif of a sequence approximating to Gly-X-Gly-X-X-Gly followed by a Lys $13-19$ residues downstream which is reactive to affinity-labelling ATP analogues (Hunter \& Cooper, 1985; Hunter, 1987). The only candidate for such a motif in the aminoglycoside phosphotransferase sequences corresponds to positions 50-55 of the Fig. 4 alignment. Here Gly is preferred at positions 50 in the APH(6)s, 52 in the APH( $\left.3^{\prime}\right)$ s and hygromycin B phosphotransferase, and 55 in all the enzymes. There is also a competely conserved Lys at an appropriate position, no. 74. This glycine-rich region is preceded by one predicted to adopt a beta-strand conformation, containing residues with beta-strandforming propensity such as Val, Thr, Trp, Tyr and Leu, as it is in other ATP-binding enzymes (Walker et al., 1982) and NAD/FAD dehydrogenases.

(ii) The characteristic Asp-Phe-Gly loop of the protein kinases (Hunter \& Cooper, 1985) may correspond to the Asp-Phe-Thr/Asp-Val-Gly/Asp-Leu-Gly/Asp-Cys-Gly in the kanamycin/

Fig. 4 (on facing page). Computer alignment of antibiotic phosphotransferases. In vertical order: APH(6), Streptomyces griseus; APH(6), S. glaucescens; APH(6)(?), Tn5; hygromycin B phosphotransferase, S. hygroscopicus; APH(3'), S. fradiae; APH(3'), Tn5; APH(3'), Tn903; APH(3'), B. circulans, and $\mathrm{APH}\left(3^{\prime}\right)$, Staphylococcus aureus. For references to these sequences, see text. Bold lower-case letters denote homologies within the $\mathrm{APH}(6)$ or $\mathrm{APH}\left(3^{\prime}\right)$ sequences only (homologies involving only the first two are omitted as trivial). All other homologies, as described in the following, are indicated by capitals. Vertical lines between the hygromycin B phosphotransferase and either APH(6) or APH( $\left.3^{\prime}\right)$ sequences, or both, indicate homologies involving the hygromycin B phosphotransferase. A vertical line above the set of sequences indicates homology between the APH(6) and $\mathrm{APH}\left(3^{\prime}\right)$ sequences, possibly but not necessarily including hygromycin B phosphotransferase. An asterisk above the set of sequences, with the letter in bold type, indicates an identical amino acid in all nine sequences. The proposed homologous blocks A1-A2, B, C1-C2, D and E1-E2 are outlined. The vertical dashed line six residues into $B$ delimits the hypothesized nucleotide-binding site (see text). The bars, numbered $1-5$, underneath the set of sequences indicate the 7-17-residue subsequences used to construct matrices employed to probe the OWL composite sequence database. 
neomycin and hygromycin B phosphotransferases at positions 249-251 of Fig. 4. The streptomycin phosphotransferases apparently have here a loop of different structure, Asp-Pro, with one less residue. On the basis of this correlation, the autophosphorylation site of tyrosine protein kinases corresponds to position 259 of the Fig. 4 alignment (Hunter \& Cooper, 1985); this residue is Tyr in some of the kanamycin/neomycin phosphotransferases.

The five sequences used by us to probe the database relate as follows to the sequences suggested by Heinzel et al. (1988) and Brenner (1987) as showing homology between antibiotic phosphotransferases and protein kinases. Region I of Heinzel et al. (1988) is equivalent to our subsequence 2 plus the first five residues of our blocks $\mathrm{Cl} / 2$; their region II to our subsequence 3 but starting five residues earlier; and their region III to our subsequence 4 . Brenner's region of homology starts one residue before our subsequence 3 and extends through and beyond our subsequences 4 and 5 ( and region III of Heinzel et al., 1988). We did not use to probe the database the subsequence corresponding to region $G / K$ of Heinzel et al. (1988), namely the extreme $\mathrm{N}$-terminal part of block $\mathrm{B}$, because its distant similarity to a nucleoside-triphosphatebinding motif (see above) suggested to us that this might be relatively undiscriminating. The conservation of subsequence 3 , commented on by all three groups, may indicate a continuing similarity of function. However, confirmation of this will probably require structural studies, which may also clarify the significance in the homologies postulated between aminoglycoside phosphotransferases and protein kinases that have been discerned in other parts of these sequences. The results of our database search described above suggest to us that the extent of homology involving subsequence 3 is of a different order to any other, implying that a degree of caution might be appropriate.

It is tempting to speculate on the evolutionary implications of the antibiotic phosphotransferase/protein kinase homology. If all organisms evolved from a common pro/ eukaryote/archaebacterial ancestor (Woese, 1987), an ancestral gene may have left descendants in all eukaryotes but died out in most branches of the prokaryote line. A similar explanation could be applied to the existence of secreted calmodulin-stimulated adenyl cyclases involved in pathogenicity in Bordetella pertussis and Bacillus anthracis (Masure et al., 1987), and also of a calmodulin-like protein in the actinomycete Saccharopolyspora erythraea (Swan et al., 1987) although the function in the latter case is unknown. It is noteworthy that in these instances the functions generally lost in prokaryotes involve 'chemical messenger' systems by which events at or near the cell periphery can exert an influence in the cytoplasmic interior or the nucleus - in the evolution of prokaryotes these may have become unnecessary. It is also intriguing that both examples involve streptomycetes. If the hypothesis is accepted whereby antibiotic resistances originated in producing organisms and spread by horizontal transfer to other prokaryotes, then the antibiotic phosphotransferase/protein kinase homology joins other cases - including the three-dimensional structure of serine proteases (Hartley, 1974), the primary structure of $\alpha$ amylases (Long et al., 1987) and the presence of inositol-containing phospholipids (Schlegel, 1985) - where streptomycetes (and more generally the group extending from the corynebacteria to the actinomycetes) show resemblances to eukaryotes rather than to other prokaryotes.

We thank J. McClellan, J. McChesney and A. Mountain for advice and suggestions, and W. Piepersberg for communicating results before publication. C.-K. L. acknowledges support and leave of absence from the National University of Singapore. This work was partly funded by MRC grants 84/09250CB and 85/04672CB to S. B. Some of the sequencing was carried out in the laboratory of Dr Sydney Brenner, MRC LMB Cambridge, UK, and we thank him for the invitation and Dr Derek Hood for advice. Computer methods were developed with support from MRC grant $84 / 01410$ CB to J.C.W. We gratefully acknowledge the assistance of Alan Bleasby, Nigel Dix and Jason Swift in the computer work.

\section{REFERENCES}

Akrigg, D., Bleasby, A. J., Dix, N. I. M., Findlay, J. B. C., North, A. C. T., Parry-Smith, D., Wootton, J. C., Blundell, T. L., Gardner, S. P., Hayes, F., Islam, S., Sternberg, M. J. E., ThorN-
TON, J. M., TiCKLE, I. J. \& MurRay-Rust, P. (1988). A protein sequence/structure database. Nature, London 335, 745.

Bibb, M. J., Bibb, M. J., WARD, J. M. \& Cohen, S. N. 
(1985). Nucleotide sequence encoding and promoting expression of three antibiotic resistance genes indigenous to Streptomyces. Molecular and General Genetics 199, 26-36.

Bleasby, A. J. \& Wootton, J. C. (1989). Construction of validated, non-redundant composite protein sequence databases. Protein Engineering (in the Press).

BRENNER, S. (1987). Phosphotransferase sequence homology. Nature, London 329, 21.

Davison, M. D., Baron, M. D., Critchley, D. R. \& WoOtToN, J. C. (1989). Structural analysis of homologous repeated domains in alpha-actinin and spectrin. International Journal of Biological Macromolecules 11, 81-90.

Distler, J. \& Piepersberg, W. (1985). Cloning and characterisation of a gene from Streptomyces griseus coding for a streptomycin-phosphorylating activity. FEMS Microbiology Letters 28, 113-117.

Distler, J., Mansouri, K. \& Piepersberg, W. (1985). Streptomycin biosynthesis in Streptomyces griseus II. Adjacent genomic location of biosynthetic genes and one of two streptomycin resistance genes. FEMS Microbiology Letters 30, 151-154.

Distler, J., Braun, C., Ebert, A. \& Piepersberg, W. $(1987 a)$. Gene cluster for streptomycin biosynthesis in Streptomyces griseus: analysis of a central region including the major resistance gene. Molecular and General Genetics 208, 204-210.

Distler, J., Ebert, A., Mansouri, K., Pissowotzki, K., Stockmann, M. \& Piepersberg, W. (1987b). Gene cluster for streptomycin biosynthesis in Streptomyces griseus: nucleotide sequence of three genes and analysis of transcriptional activity. Nucleic Acids Research 15, 8041-8056.

Drummond, M. \& WootTon, J. C. (1987). Sequence of nifL from Klebsiella pneumoniae: mode of action and relationship to two families of regulatory proteins. Molecular Microbiology 1, 37-44.

Eliopoulos, E., Geddes, A. J., Brett, M., Pappin, D. J. C. \& FindLAY, J. B. C. (1982). A structural model for the chromophore-binding region of ovine rhodopsin. International Journal of Biological Macromolecules 4, 263-268.

Ferretti, J. J., Gilmore, K. S. \& Courvalin, P. (1986). Nucleotide sequence analysis of the gene specifying the bifunctional 6 '-aminoglycoside acetyltransferase 2"-aminoglycoside phosphotransferase enzyme in Streptococcus faecalis and identification and cloning of gene regions specifying the two activities. Journal of Bacteriology 167, 631-638.

GIL, J. A. \& HopwooD, D. A. (1983). Cloning and expression of a $p$-aminobenzoic acid synthetase gene of the candicidin producer, Streptomyces griseus. Gene 25, 119-132.

GIL, J. A., Kieser, H. M. \& Hopwood, D. A. (1985). Cloning of a chloramphenicol acetyltransferase gene of Streptomyces acrimycini and its expression in Streptomyces lividans and Escherichia coli. Gene 38, 1-8.

HaRTLEY, B. S. (1974). Enzyme families. In Evolution in the Microbial World (24th Symposium of the Society for General Microbiology), pp. 151-182. Edited by M. J. Carlile \& J. J. Skehel. Cambridge, UK: Cambridge University Press.

Heinzel, P., Werbitzky, O., Distler, J. \& Piepers-
BERG, W. (1988). A second streptomycin resistance gene from Streptomyces griseus codes for streptomycin $3^{\prime \prime}$-phosphotransferase. Relationships between antibiotic and protein kinases. Archives of Microbiology 150, 184-192.

Herbert, C. J., Sarwar, M., Ner, S. S., Giles, I. G. \& AKHTAR, M. (1986). Sequence and interspecies transfer of an aminoglycoside phosphotransferase gene (APH) of Bacillus circulans. Biochemical Journal 233, 383-393.

Hercomb, J., Thierbach, G., Baumberg, S. \& Parish, J. H. (1987). Cloning, characterization and expression in Escherichia coli of a leucine biosynthetic gene from Streptomyces rochei. Journal of General Microbiology 133, 317-322.

hintermanN, G., Crameri, R., Vögtli, M. \& HütTER, R. (1984). Streptomycin-sensitivity in Streptomyces glaucescens is due to deletions comprising the structural gene coding for a specific phosphotransferase. Molecular and General Genetics 196, 513-520.

Hopwood, D. A., BibB, M. J., Chater, K. F., Janssen, G. R., Malpartida, F. \& SMith, C. P. (1986). Regulation of gene expression in antibiotic-producing Streptomyces. In Regulation of Gene Expression25 Years On (39th Symposium of the Society for General Microbiology), pp. 251-276. Edited by I. R. Booth \& C. F. Higgins. Cambridge, UK : Cambridge University Press.

Hunter, I. S. \& BaUmberG, S. (1989). Molecular genetics of antibiotic formation. In Microbial Products: New Approaches (44th Symposium of the Society for General Microbiology), pp. 121-162. Edited by S. Baumberg, I. S. Hunter \& P. M. Rhodes. Cambridge, UK: Cambridge University Press.

Hunter, T. (1987). A thousand and one protein kinases. Cell 50, 823-829.

Hunter, T. \& CoOPER, J. A. (1985). Protein-tyrosine kinases. Annual Review of Biochemistry 54, 897-930.

IsH-Horowicz, D. \& BURKE, J. F. (1981). Rapid and efficient cosmid cloning. Nucleic Acids Research 9, 2989-2998.

KIESER, T. (1984). Factors affecting the isolation of cccDNA from Streptomyces lividans and Escherichia coli. Plasmid 12, 19-36.

LAEMMLI, U. K. (1970). Cleavage of structural proteins during the assembly of the head of bacteriophage T4. Nature, London 227, 680-685.

Long, C. M., Virolle, M.-J., Chang, S.-Y., Chang, S. \& BIBB, M. J. (1987). $\alpha$-Amylase gene of Streptomyces limosus: nucleotide sequence, expression motifs, and amino acid sequence homology to mammalian and invertebrate $\alpha$-amylases. Journal of Bacteriology 169, 5745-5754.

Maniatis, T., Fritsch, E. F. \& SAmbrook, J. (1982). Molecular Cloning. A Laboratory Manual. Cold Spring Harbor, NY: Cold Spring Harbor Laboratory.

Mann, N. H., Mountain, A., Munton, R. N., Smith, M. C. M. \& BAUmberg, S. 1984). Transcription analysis of a Bacillus subtilis arg gene following cloning in Escherichia coli in an initially unstable hybrid plasmid. Molecular and General Genetics 197, 75-81.

Masure, H. R., Shattuck, R. L. \& Storm, D. R. (1987). Mechanisms of bacterial pathogenicity that 
involve production of calmodulin-sensitive adenylate cyclases. Microbiological Reviews 51, 60-65.

Nichols, B. P. \& YANOFSKY, C. (1983). Plasmids containing the trp promoters of $E$. coli and Serratia marcescens and their use in expressing cloned genes. Methods in Enzymology 101, 155-164.

OHNuki, T., Imanaka, T. \& AIBA, S. (1985). Selfcloning in Streptomyces griseus of a str gene cluster for streptomycin biosynthesis and streptomycin resistance. Journal of Bacteriology 164, 85-94.

Piepersberg, W., Distler, J., Heinzel, P. \& PerezGonZalez, J.-A. (1988). Antibiotic resistance by modification: many resistance genes could be derived from cellular control genes in actinomycetes. - A hypothesis. Actinomycetologia 2, 83-98.

REEVE, J. N. (1979). Use of minicells for bacteriophage-directed polypeptide synthesis. Methods in Enzymology 68, 493-503.

SchechtMAN, M. G. \& YANOFSKY, C. (1983). Structure of the tri-functional trp- 1 gene from Neurospora crassa and its aberrant expression in Escherichia coli. Journal of Molecular and Applied Genetics 2, 83-89.

SCHLEGEL, H.-G. (1985). General Microbiology, 6th edn. Cambridge, UK : Cambridge University Press.

Shinkawa, H., Sugiyama, M., Nimi, O. \& Nomi, R. (1985). Molecular cloning and expression in Streptomyces lividans of a streptomycin-6-phosphotransferase gene from a streptomycin-producing microorganism. FEBS Letters 181, 385-389.

Shinkawa, H., Sugiyama, M. \& Nimi, O. (1987). The nucleotide sequence of a streptomycin 6-phosphotransferase gene from a streptomycin producer. Journal of General Microbiology 133, 1289-1296.

Shinkawa, H., Sugiyama, M. \& Nimi, O. (1988). The nucleotide sequence of a streptomycin 6-phosphotransferase gene from a streptomycin producer (corrigendum). Journal of General Microbiology 134, 1391-1394.

Smith, M. C. M., Mountain, A. \& Baumberg, S.
(1986). Sequence analysis of the Bacillus subtilis $\arg C$ promoter. Gene 49, 53-60.

SMITH, T. F. \& WATERMAN, M. S. (1981). Identification of common molecular sequences. Journal of Molecular Biology 147, 195-197.

Tohyama, H., Shigyo, T. \& OKami, Y. (1984). Cloning of streptomycin resistance gene from a streptomycin producing streptomycete. Journal of Antibiotics 37, 1736-1737.

Tohyama, H., Okami, Y. \& Umezawa, H. (1987). Nucleotide sequence of the streptomycin phosphotransferase and amidinotransferase genes from Streptomyces griseus. Nucleic Acids Research 15, 1819-1833.

VAllins, W. J. S. \& Baumberg, S. (1985). Cloning of a DNA fragment from Streptomyces griseus which directs streptomycin phosphotransferase activity. Journal of General Microbiology 131, 1657-1669.

VöGTLI, M. \& HÜTTER, R. (1987). Characterisation of the hydroxystreptomycin phosphotransferase gene (sph) of Streptomyces glaucescens: nucleotide sequence and promoter analysis. Molecular and General Genetics 208, 195-203.

Walker, J. E., SARAste, M., Runswick, M. J. \& Gay, N. J. (1982). Distantly related sequences in the $\alpha$ and $\beta$-subunits of ATP synthase, myosin, kinases and other ATP-requiring enzymes and a common nucleotide fold. EMBO Journal 1, 945-951.

WOESE, C. R. (1987). Bacterial evolution. Microbiological Reviews 51, 221-271.

Wootton, J. C. \& DRummond, M. H. (1989). The Qlinker: a class of interdomain sequences found in bacterial multidomain regulatory proteins. Protein Engineering 2, 535-543.

Zalacain, M., Gonzales, A., Guerrero, M. C., Mattaliano, F., Malpartida, F. \& Jimenez, A. (1986). Nucleotide sequence of the hygromycin B phosphotransferase gene from Streptomyces hygroscopicus. Nucleic Acids Research 14, 1565-1581. 